\title{
Força muscular e funcionalidade de pacientes hospitalizados admitidos por afecções respiratórias
}

\author{
Muscle strength and functionality of hospitalized patients admitted for respiratory affections \\ Fuerza y funcionalidad muscular de pacientes hospitalizados admitidos por enfermedades \\ respiratorias
}

Recebido: 28/10/2021 | Revisado: 05/11/2021 | Aceito: 08/11/2021 | Publicado: 13/11/2021

\author{
Gabriel Cardoso Santos \\ ORCID: https://orcid.org/0000-0003-3707-8509 \\ Universidade Federal de Sergipe, Brasil \\ E-mail: cstosgabriel17@gmail.com \\ Ione Carla dos Santos \\ ORCID: https://orcid.org/0000-0002-4657-6959 \\ Universidade Federal de Sergipe, Brasil \\ E-mail: ione_forever@hotmail.com \\ Grazielle Batista dos Santos \\ ORCID: https://orcid.org/0000-0001-5074-1980 \\ Universidade Federal de Sergipe, Brasil \\ E-mail: graziellebatista2014@gmail.com \\ Juarez Alexandre Oliveira Ferreira \\ ORCID: https://orcid.org/0000-0002-6454-7692 \\ Universidade Federal de Sergipe, Brasil \\ E-mail: juarez_alexandre17@live.com \\ Lucas Aragão da Hora Almeida \\ ORCID: https://orcid.org/0000-0002-1748-4086 \\ Empresa Brasileira de Serviços Hospitalares, Brasil \\ E-mail: biolucasaragao@gmail.com \\ Carlos José Oliveira de Matos \\ ORCID: https://orcid.org/0000-0002-2168-7839 \\ Universidade Federal de Sergipe, Brasil \\ E-mail: cjomatos@yahoo.com.br \\ Érika Ramos Silva \\ ORCID: https://orcid.org/0000-0002-0634-976X \\ Universidade Federal de Sergipe, Brasil \\ E-mail: erikarns@academico.ufs.br
}

\begin{abstract}
Resumo
Objetivo: Analisar a força muscular e a funcionalidade de pacientes hospitalizados admitidos por afecções respiratórias. Metodologia: Estudo transversal, realizado em pacientes diagnosticados e internados com doenças respiratórias. As avaliações foram realizadas por meio da Medical Research Council (MRC), Medida de independência funcional (MIF), World Health Disability Assessment Schedule (WHODAS), manovacuometria e dinamometria em pacientes internados no Hospital Universitário de Lagarto. Resultados: Participaram do respectivo estudo 97 pacientes, onde observou-se redução das forças musculares periféricas e respiratórias. Ao analisar a funcionalidade, notou-se moderada dificuldade funcional, sendo ambos instrumentos correlatos $(r=-722 ; p<0,01)$. Ao analisar a relação entre média de MRC (MMSS) e FPP D e E, demostrou-se que houve correlação alta e positiva $(\mathrm{p}<0,01)$ entre as mesmas. Notou-se uma correlação alta e positiva $(\mathrm{p}<0,01)$ entre MRC e MIF, e alta e negativa com o WHODAS ( $\mathrm{r}=-598 ; \mathrm{p}<0,01)$. Ao avaliar as médias da FPP e do MRC entre os grupos acima de 60 anos e abaixo de 60 anos, verificou-se que o fator idade contribui para a perda da força muscular e, consequentemente, da funcionalidade $(\mathrm{p}=0.000)$. Conclusão: Os pacientes internados por afecções respiratórias agudas ou crônicas agudizadas apresentaram redução da força muscular periférica e respiratória. Esta realidade ressalta a importância de uma assistência fisioterapêutica precoce, pautada na funcionalidade e no ganho de força muscular desses pacientes.

Palavras-chave: Força muscular; Doenças respiratórias; Hospitalização.
\end{abstract}

\section{Abstract}

Objective: To analyze the muscle strength and functionality of hospitalized patients admitted for respiratory conditions. Methodology: Cross-sectional study, carried out in patients diagnosed and hospitalized with respiratory diseases. The assessments were performed using the Medical Research Council (MRC), Functional Independence Measure (MIF), World Health Disability Assessment Schedule (WHODAS), manovacuometry and dynamometry in 
patients admitted to Hospital University Student of Lagarto. Results: 97 patients participated in the respective study, where a reduction in peripheral and respiratory muscle strength was observed. When analyzing functionality, moderate functional difficulty was noted, both instruments being correlated $(r=-722 ; p<0.01)$. By analyzing the relationship between mean MRC (MMSS) and Palmar Grip Force D and E, it was demonstrated that there was a high and positive correlation $(\mathrm{p}<0.01)$ between them. There was a high positive correlation $(\mathrm{p}<0.01)$ between MRC and MIF, and a high and negative correlation with WHODAS $(r=-598 ; \mathrm{p}<0.01)$. When evaluating the averages of Palmar Grip Force and MRC between the groups over 60 years and under 60 years, it was found that the age factor contributes to the loss of muscle strength and, consequently, of functionality $(\mathrm{p}=0.000)$. Conclusion: Patients hospitalized due to acute or acute chronic respiratory diseases presented reduced peripheral and respiratory muscle strength. This reality highlights the importance of early physical therapy assistance, based on functionality and muscle strength gain in these patients.

Keywords: Muscle strength; Respiratory tract diseases; Hospitalization.

\begin{abstract}
Resumen
Objetivo: Analizar la fuerza muscular y la funcionalidad de los pacientes hospitalizados ingresados por afecciones respiratorias. Metodologia: estudio transversal, realizado en pacientes diagnosticados y hospitalizados de enfermedades respiratorias. Las evaluaciones se realizaron mediante el Medical Research Council (MRC), Functional Independence Measure (MIF), World Health Disability Assessment Schedule (WHODAS), manovacuometría y dinamometría en pacientes ingresados en el Hospital Universitário de Lagarto. Resultados: 97 pacientes participaron en el estudio respectivo, donde se observó una reducción de la fuerza de los músculos periféricos y respiratorios. Al analizar la funcionalidad se observó dificultad funcional moderada, correlacionándose ambos instrumentos $(\mathrm{r}=-722 ; \mathrm{p}$ $<0,01)$. Al analizar la relación entre la media de MRC (MMSS) y FPP D y E, se demostró que existía una correlación alta y positiva ( $\mathrm{p}<0.01)$ entre ellos. Hubo una alta correlación positiva $(\mathrm{p}<0.01)$ entre MRC y MIF, y una alta y negativa correlación con WHODAS ( $\mathrm{r}=-598$; $\mathrm{p}<0.01$ ). Al evaluar los promedios de FPP y MRC entre los grupos mayores de 60 años y menores de 60 años, se encontró que el factor edad contribuye a la pérdida de fuerza muscular $\mathrm{y}$, en consecuencia, de funcionalidad $(\mathrm{p}=0,000)$. Conclusión: Los pacientes hospitalizados por enfermedades respiratorias agudas o crónicas agudas presentaron disminución de la fuerza de los músculos periféricos y respiratorios. Esta realidad resalta la importancia de la asistencia de fisioterapia temprana, basada en la funcionalidad y la ganancia de fuerza muscular en estos pacientes.
\end{abstract}

Palabras clave: Fuerza muscular; Enfermedades respiratorias; Hospitalización.

\title{
1. Introdução
}

O processo de hospitalização resulta em declínio funcional, e está associado ao pior prognóstico do paciente. Além disso, a perda da funcionalidade está relacionada a maior utilização dos serviços de saúde e a custos sociais mais elevados, resultando em implicações para o sistema de saúde (Basic et al., 2017). Entre as causas de hospitalização, as doenças respiratórias agudas (DRA’s) têm destaque mundial e por isto são encaradas como importante problema de saúde pública. A elevada incidência, os altos custos com tratamento de saúde, as possíveis complicações decorrentes das comorbidades associadas, perdas funcionais (dependência), redução da mobilidade e comprometimento da cognição, com elevados índices de morbimortalidade, são observados tanto em países subdesenvolvidos, quanto em países desenvolvidos (Romanholi-Cória et al., 2017).

De modo geral, as internações hospitalares são caracterizadas pela predominância de pacientes idosos, com morbidades respiratórias e cardiovasculares crônicas (Morosini et al., 2011; Lima-Costa; Loyola-Filho; Matos, 2007). No Brasil, cerca de 19,5\% dos óbitos durante as internações, são justificados pelas complicações respiratórias associadas a longos períodos de internação hospitalar, incapacidades provocadas ou exacerbadas pela imobilidade e restrição ao leito, formação de úlceras de pressão e maior exposição às infecções hospitalares (Santos, 2007).

A perda da função muscular é uma das primeiras consequências do período de internação. A mesma é caracterizada por uma fraqueza muscular adquirida (FMA), difusa e simétrica que envolve a musculatura dos membros superiores e inferiores. No paciente hospitalizado, uma das consequências da FMA é a alteração na funcionalidade, que consiste na redução da capacidade de realizar suas atividades entre o período prévio à internação e durante a estadia hospitalar, resultando em diminuição dos desempenhos físicos e cognitivos (Ferreira et al., 2018). A imobilização prolongada também gera rápida atrofia 
muscular, com comprometimento muscular tempo dependente. A perda de massa muscular é mais intensa na fase inicial da imobilização, tendo atenuação ao longo do tempo (Ferreira et al., 2004).

O perfil dos pacientes hospitalizados por afecções respiratórias não é totalmente conhecido no município de Lagarto, Sergipe. Da mesma forma que também não são conhecidos programas de atenção integral a este grupo de pacientes, com fins de prevenir piora funcional antes e após a internação hospitalar. Com base nesta realidade, é necessário caracterizar os pacientes internados com doenças respiratórias, com fins de identificar suas necessidades, visando o aperfeiçoamento e qualidade dos cuidados prestados em saúde.

Portanto, este estudo tem como objetivo analisar o perfil clínico, a força muscular e a funcionalidade de pacientes admitidos por afecções respiratórias agudas ou crônicas agudizadas.

\section{Metodologia}

Trata-se de uma pesquisa de caráter transversal, que foi realizada no Hospital Universitário de Lagarto, em Sergipe, aprovada pelo Comitê de Ética e Pesquisa sob o número de CAAE: 87234818.4.0000.5546 e Parecer: 2.724.602.

O período de coleta ocorreu entre os meses de março e setembro de 2019 e incluiu os pacientes admitidos no hospital por diagnóstico clínico de doenças respiratórias agudas ou crônicas agudizadas, de ambos os sexos, com idade a partir de 18 anos, nível de cognição que permitisse adequada participação nos testes e avaliações que foram realizadas, concordância em participar da pesquisa e que não apresentassem afecções neurológicas limitantes, sendo a amostra desta pesquisa selecionada por conveniência. As avaliações aconteceram no período inicial da internação, em intervalo de tempo compreendido entre $24 \mathrm{~h}$ e 72h após a data de admissão hospitalar.

Foram excluídos aqueles que: utilizassem via aérea artificial (tubos endotraqueais); apresentassem instabilidade clínica ou hemodinâmica ou que, por algum motivo, não pudessem realizar um dos testes ou análises aqui propostas. As avaliações foram norteadas por uma ficha de avaliação, que serviu para registro das variáveis pesquisadas.

Para avaliar a força muscular periférica, foram utilizados 02 instrumentos de medida. O Medical Research Council (MRC) e o dinamômetro (handgrip) para medida da força de preensão palmar. O Medical Research Council (MRC) é uma ferramenta avaliativa amplamente utilizada para diagnóstico clínico e funcional da força muscular neste perfil de pacientes. O escore do MRC utiliza variáveis categóricas ordinais que variam de 0 (sem contração nenhuma) a 5 (força muscular normal com resistência), tendo pontuação mínima igual a 0 e máxima igual a 60. São avaliados cerca de 12 grupos musculares (abdutores de ombro, flexores de cotovelo, extensores de punho, flexores de quadril, extensores de joelho e dorsiflexores), avaliados bilateralmente. Os voluntários foram posicionados sentados com o quadril e joelhos flexionados à $90^{\circ}$. A fim de melhor padronizar a resistência manual oferecida, o avaliador classificou como grau 4 aquele paciente cuja força gerada, vencesse a resistência imposta por 2 dedos e como grau 5 aquele paciente que vencesse a resistência imposta por quatro dedos (Martinez et al., 2015).

Para avaliação do pico de força de preensão palmar, foi utilizado o dinamômetro (handgrip), um método sensível e mais preciso para quantificar mudanças de força de preensão palmar (FPP) ao longo do tempo, particularmente em pacientes com força suficiente para vencer a gravidade (Vieira et al., 2018).

Foi considerado o maior valor obtido a partir de três medidas, intervaladas por dois minutos de descanso. Caso houvesse discrepância entre os valores obtidos, nova medida era realizada. Os pacientes executaram o teste com o membro superior dominante e não dominante em posição sentada com o cotovelo flexionado em um ângulo de $90^{\circ}$, antebraço e pulso em posição neutra, de acordo com as recomendações da Sociedade Brasileira de Terapeutas da Mão (SBTM) (Figueiredo et al., 2007). 
Para a avaliação das pressões respiratórias máximas foi utilizado o manovacuômetro, que é um medidor de pressões respiratórias negativas (pressão inspiratória máxima, Pimáx) e positivas (pressão expiratória máxima, Pemáx). Então, os pacientes foram posicionados em sedestação com flexão de quadris e joelhos à $90^{\circ}$, com flexão anterior do tronco, sendo orientados sobre como realizar o procedimento. Foi considerado o maior valor obtido a partir de três medidas, intervaladas por dois minutos de descanso. Caso houvesse discrepância entre os valores obtidos, nova medida era realizada (Volianitis et al., 2001).

Quanto a obtenção da Pimáx, a inspiração se iniciou a partir do Volume Residual (VR), sendo estimulada a sustentação da máxima inspiração por pelo menos 2 segundos. Já para a mensuração da Pemáx, o voluntário começou no nível da Capacidade Pulmonar Total (CPT), isto é, após uma inspiração profunda e, então, realizou expiração forçada sustentando a pressão por 2 segundos e evitando "bochechos" ou escapes aéreos. Para cálculo dos valores preditos, foi utilizada a fórmula preconizada por Neder et al., (1999).

HOMENS: Pimáx $=-0,80$ (idade) +155.3 . Pemáx: -0.81 (idade) +165.3 .
MULHERES: Pimáx $=-0.49$ (idade) +100.4 . Pemáx: -0.61 (idade $)+115.6$.

Quanto a análise da funcionalidade, foi utilizada a escala de Medida de Independência Funcional e o World Health Disability Assessment Schedule 2.0 (WHODAS 2.0). A escala de medida de independência funcional (MIF) é um instrumento amplamente utilizado em hospitais ou em unidades de terapia intensiva com o objetivo de acompanhar pessoas que estão em um processo de reabilitação. Ela contém 18 itens agrupados em seis dimensões: autocuidado, locomoção, transferência, comunicação, controle dos esfíncteres e cognição social. Cada dimensão é avaliada pela soma dos itens que a compõem, o escore total da MIF pode variar de 18 a 126 pontos. Os níveis de dependência são classificados de acordo com o escore total da MIF, onde: 18 indica dependência completa; 19 a 60, dependência modificada (assistência de até 50\% das tarefas); 61 a 103, dependência modificada (com assistência nas tarefas de até 25\%) e 104 a 126, indicam independência completa/modificada (Assis et al., 2015).

Além do nível de independência, o componente cognição social foi analisado separadamente com fins de excluir os pacientes que apresentassem déficit cognitivo mais comprometido. Aqueles que apresentassem valores a partir de 21 pontos, foram inclusos na pesquisa.

Já o WHODAS 2.0, é um instrumento desenvolvido a partir de um conjunto de itens da CIF, validado e adaptado culturalmente para o Brasil, que pode ser aplicado a diferentes populações tanto em âmbito clínico, para medir o impacto de uma dada intervenção, quanto no âmbito populacional em estudos epidemiológicos (Organização Mundial da Saúde, 2015; Federici et al., 2017).

O questionário possui seis domínios que remetem à CIF, sendo eles: cognição (pontuação máxima 30 pontos), mobilidade (pontuação máxima 25 pontos), autocuidado (pontuação máxima 20 pontos), relações interpessoais (pontuação máxima 25 pontos), atividades de vida (pontuação máxima 40 pontos) e participação (pontuação máxima 40 pontos), total do questionário 180 pontos. Para a contagem dos escores de cada domínio é atribuído o valor 1 para nenhuma incapacidade, 2 para ligeira incapacidade, 3 para incapacidade moderada, 4 para incapacidade grave e 5 para incapacidade muito grave (Silva et al., 2016; Chalmers et al., 2010). Para análise do score total do WHODAS 2.0, foi estipulado ponto de corte em porcentagem: 0-4\% (nenhuma dificuldade) 5-24\% (dificuldade ligeira), 25-49\% (dificuldade moderada), 50-95\% (dificuldade grave), 96-100\% (dificuldade completa/não faz) (Chalmers et al., 2010). 


\subsection{Análise dos dados}

As variáveis numéricas foram testadas quanto à distribuição de normalidade por meio do teste Shapiro-Wilk. Os dados normais foram apresentados em média e desvio padrão. Para as comparações entre os grupos, foi utilizado o teste de T de Student e para as correlações o Teste de Pearson. O teste qui quadrado foi utilizado para as demais comparações. A significância estatística foi estipulada em $95 \%(\mathrm{p} \leq 0,05)$. Para todas as análises, foi utilizado o software IBM ${ }^{\circledR}$ SPSS Statistical Package for the Social Sciences 24.0 Mac (SPSS 24.0 Mac, SPSS Inc., Chicago, Illinois, EUA).

\section{Resultados}

\section{Caracterização da amostra}

A pesquisa incluiu 97 voluntários. Destes, 43,8\% ( $n=42)$ eram do sexo masculino e 56,3\% ( $n=55)$ do sexo feminino, com média de idade foi de 66,6 $( \pm 16,5)$ anos. Em sua maioria eram sedentários $(90,6 \%, n=87)$ e fumantes $(39,6 \%, n=38)$. Entre o público de fumantes $(60,5 \%, \mathrm{n}=23)$ eram do sexo feminino e $(39,4 \%, \mathrm{n}=15)$ do sexo masculino.

Entre as afecções respiratórias, que justificaram o internamento hospitalar, houve prevalência de Pneumonia (57,3\%, $\mathrm{n}=55$ ), seguida de Doença Pulmonar Obstrutiva Crônica exacerbada (DPOC) com (20,8\%, n= 21), Edema Agudo Pulmonar $(10,4 \%, n=11)$, Asma $(3,1 \%, n=3)$, Insuficiência Respiratória Aguda (IRPA) e Derrame Pleural $(2,1 \%, n=2)$, Pneumotórax $(2 \%, \mathrm{n}=2)$, finalizando com Tuberculose $(1 \%, \mathrm{n}=1)$ (Tabela 1$)$.

Tabela 1 - Principais causas de internações por afecções respiratórias.

\begin{tabular}{ccc}
\hline & Frequência & Percentual \\
\hline Pneumonia & 55 & 57.3 \\
DPOC & 21 & 20.8 \\
Edema Agudo Pulmonar & 11 & 10.4 \\
Asma & 3 & 3.1 \\
IRPA & 2 & 2.1 \\
Derrame Pleural & 2 & 2.1 \\
Pneumotórax & 2 & 2.0 \\
Tuberculose & 1 & 1.0 \\
\hline
\end{tabular}

DPOC: Doença Pulmonar Obstrutiva Crônica; IRPA: Insuficiência Respiratória Aguda. Fonte: Autores (2019).

Quanto às variáveis funcionais que denotam força muscular o MRC total foi cerca de 47,42 $( \pm 8,97)$ sendo a média de 24.06 ( \pm 4.679$)$ para MRC (MMSS) e 23.48 (4.470) para MRC (MMII). Em todas as medidas confere-se redução na força muscular mensurada. Valores baixos, confirmados na FPP do MS dominante, onde os destros apresentaram média de 19,36 $( \pm 8,54)$ Kgf e os canhotos, valor médio de 17,27 $( \pm 9,38)$ Kgf.

Quando mensuradas as pressões respiratórias máximas, a Pimáx obteve-se o valor médio de $-31,45( \pm 20,44) \mathrm{cmH}_{2} \mathrm{O}$, compatível com fraqueza da musculatura inspiratória e $+40.34( \pm 26.41) \mathrm{cmH}_{2} \mathrm{O}$ da Pemáx. Ao ser analisada a média de funcionalidade no WHODAS 2.0 a pontuação média e desvio padrão obtidas foi $0,32( \pm 0,26)$, denotando moderada dificuldade funcional. Na Medida de Independência Funcional (MIF) foi de 96,67 $( \pm 32,68)$, mostrando que os pacientes possuem uma dependência funcional em $25 \%$ das suas tarefas.

Quanto ao domínio mais prejudicado no WHODAS 2.0, a Atividades de Vida mostrou-se ser o item mais comprometido funcionalmente, com média e desvio padrão de $0.481( \pm 0.341)$, seguido de Participação com média 0.398 
$( \pm 0.298)$ e Mobilidade com média $0.363( \pm 0.337)$, notando-se assim, que o processo de internação leva a uma perda na funcionalidade e nas Atividades de Vida Diária (AVD's) (Tabela 2).

Tabela 2 - Domínios do WHODAS 2.0 avaliados durante o processo de hospitalização.

\begin{tabular}{ccc}
\hline & Média & Desvio Padrão \\
\hline COGNIÇÃO & 0.210 & 0.284 \\
MOBILIDADE & 0.363 & 0.337 \\
AUTO-CUIDADO & 0.287 & 0.334 \\
REL. INTERPESSOAIS & 0.181 & 0.237 \\
ATIVIDADES DE VIDA & 0.481 & 0.341 \\
PARTICIPAÇÃO & 0.398 & 0.298 \\
\hline
\end{tabular}

Ao ser analisada a média de funcionalidade no WHODAS 2.0, a pontuação média e desvio padrão obtidas foi 0,32 ( $\pm 0,26)$, denotando moderada dificuldade funcional. Fonte: Autores (2019).

Ao verificar a correlação entre as variáveis analisadas, nota-se uma correlação alta e negativa entre as variáveis Idade, MRC (TOTAL) e FPP (D e E) com ( $<<0,01)$. Nota-se também uma correlação alta e positiva entre as variáveis Idade, Pimax e WHODAS ( $p<0,01)$. Quanto a FFP D e E, houve uma correlação alta e negativa com as variáveis Pimax e WHODAS (p< 0,01), e uma correlação alta e positiva com FFP E e MRC (TOTAL) ( $<<0,01)$. Ao analisar a correlação entre a média de Pimax com MRC (TOTAL), houve uma correlação alta e negativa, e alta e positiva quando comparada ao WHODAS (p< 0,01). Notou-se uma correlação alta e negativa entre as médias do MRC (TOTAL) e do WHODAS (p< 0,01). A partir dos dados descritos acima, percebe-se que, em pacientes hospitalizados, quanto maior idade, maior será a perca funcional e menor será a força muscular, seja esta de MMSS, MMII ou da musculatura respiratória (Tabela 3).

Tabela 3 - Correlação entre as médias das variáveis Idade, FPP (D e E), Pimax, MRC (TOTAL) e WHODAS.

\begin{tabular}{|c|c|c|c|c|c|}
\hline & FPP (D) & FPP (E) & PIMAX & TOTAL(MRC) & WHODAS \\
\hline \multirow[t]{2}{*}{ IDADE } &,$- 390 * *$ & $-.335 * *$ &,$- 395 * *$ &,$- 332 * *$ &, $283 * *$ \\
\hline & .000 & .001 & .000 & .001 & .005 \\
\hline FPP (D) & &, $797 * *$ &,$- 510 * *$ &, $705 * *$ &,$- 476 * *$ \\
\hline \multirow[t]{3}{*}{ FPP (E) } & &,$- 487 * *$ & .000 & .000 & .000 \\
\hline & & .000 & &, $629 * *$ &,$- 357 * *$ \\
\hline & & & & .000 & .000 \\
\hline \multirow[t]{2}{*}{ PIMAX } & & & &,$- 444 * *$ &, $309 * *$ \\
\hline & & & & .000 & .002 \\
\hline \multirow[t]{2}{*}{ TOTAL(MRC) } & & & & &,$- 598 * *$ \\
\hline & & & & & .000 \\
\hline
\end{tabular}

* Correlação de Pearson. Valor de p $\leq$ 0,05. PiMAX: Pressão inspiratória máxima; PeMAX: Pressão expiratória máxima; MRC: Medical Research Council; MMII: Membros inferiores; MMSS: Membros superiores; FPP: Força de Preensão Palmar; MIF: Medida de Independência Funcional; WHODAS 2.0: World Health Organization Disability Assessment Schedule 2.0. Fonte: Autores (2019). 
Ao ser analisada a correlação entre as variáveis mensuradas, foi constatada alta e negativa correlação entre a variável Pimáx e as variáveis Pemáx, MRC (MMSS), FPP D e E (p< 0,01). Quanto à Pemáx houve uma correlação alta e positiva entre as variáveis MRC (MMSS) e FPP D e E (p< 0,01). Ao analisar a relação entre média de MRC (MMSS) e FPP D e E, demostrou-se que houve correlação alta e positiva $(\mathrm{p}<0,01)$ entre as mesmas. Notou-se uma correlação alta e positiva $(\mathrm{p}<$ 0,01) entre MRC (MMII) e MRC (total) com a MIF, e alta e negativa com o WHODAS ( $p<0,01)$. Ao verificar as variáveis funcionais MIF e WHODAS, demonstraram-se uma correlação alta e negativa $(p<0,01)$. Em consequência, os dados sugerem que o período de hospitalização é responsável por resultar no declínio da força muscular, seja ela periférica ou não, ocasionando assim, a perca significativa da funcionalidade, como visto principalmente na correlação realizada entre os dados de instrumentos que medem a funcionalidade e a força muscular, como a MIF, o WHODAS e o MRC, respectivamente. (Tabela 4).

Tabela 4 - Correlação entre as médias das variáveis Pimáx, PeMáx com o MRC, FPP (D e E), MIF e WHODAS.

\begin{tabular}{|c|c|c|c|c|c|c|c|c|}
\hline & PEMAX & $\begin{array}{l}\text { MRC } \\
\text { (MISS) }\end{array}$ & $\begin{array}{l}\text { MRC } \\
\text { (MMII) }\end{array}$ & $\begin{array}{l}\text { TOTAL } \\
\text { (MRC) }\end{array}$ & $\begin{array}{l}\text { FPP } \\
\text { (D) }\end{array}$ & $\begin{array}{l}\text { FPP } \\
\text { (E) }\end{array}$ & MIF & WHODAS \\
\hline \multirow[t]{2}{*}{ PIMAX } &,$- 703^{* *}$ &,$- 458^{* \cdots}$ & -122 & -.079 &,$- 510^{* *}$ &,$- 487^{* *}$ & .033 & -.013 \\
\hline & .000 & .000 & .235 & .443 & .000 & .000 & .750 & .902 \\
\hline \multirow[t]{2}{*}{ PEMAX } & &, $441^{* *}$ & .108 & .096 &, $592^{* *}$ &, $507^{* *}$ & .063 & .025 \\
\hline & & .000 & .294 & .351 & .000 & .000 & .545 & .808 \\
\hline \multirow[t]{2}{*}{ MRC (MMSS) } & & & -.054 & -.070 &, $697^{* *}$ &, $614^{* *}$ & -.103 & .076 \\
\hline & & & .599 & .496 & .000 & .000 & .317 & .461 \\
\hline \multirow{2}{*}{$\begin{array}{c}\text { MRC } \\
\text { (MIII) }\end{array}$} & & & &, $951^{* *}$ & .065 & .118 &, $642^{* *}$ &,$- 564^{* *}$ \\
\hline & & & & .000 & .527 & .254 & .000 & .000 \\
\hline \multirow{2}{*}{$\begin{array}{l}\text { TOTAL } \\
\text { (MRC) }\end{array}$} & & & & & .029 & .096 &, $681^{* x}$ &,$- 598^{* x}$ \\
\hline & & & & & .781 & .354 & .000 & .000 \\
\hline \multirow{2}{*}{$\begin{array}{l}\text { FPP } \\
\text { (D) }\end{array}$} & & & & & &, $797^{* *}$ & .007 & -.005 \\
\hline & & & & & & .000 & .946 & .960 \\
\hline \multirow{2}{*}{$\begin{array}{l}\text { FPP } \\
\text { (E) }\end{array}$} & & & & & & & .013 & .002 \\
\hline & & & & & & & .902 & .984 \\
\hline \multirow[t]{2}{*}{ MIF } & & & & & & & &,$- 722^{* *}$ \\
\hline & & & & & & & & .000 \\
\hline
\end{tabular}

* Correlação de Pearson. Valor de $\mathrm{p} \leq$ 0,05. PiMAX: Pressão inspiratória máxima; PeMAX: Pressão expiratória máxima; MRC: Medical Research Council; MMII: Membros inferiores; MMSS: Membros superiores; FPP: Força de Preensão Palmar; MIF: Medida de Independência Funcional; WHODAS 2.0: World Health Organization Disability Assessment Schedule 2.0. Fonte: Autores (2019).

Ao comparar as médias de FPP D e E, MRC (MMSS, MMII e Total) houve diferença entre os grupos acima de 60 anos e abaixo de 60 anos ( $\mathrm{p}=0.000)$, demonstrando que nos grupos de maior idade a força periférica foi menor. Quando comparados os valores médios de MRC Total, de MMSS e MMII, os valores semelhantes que denotam fraqueza demonstram que a perda de força é geral, tanto em segmentos superiores quanto inferiores (Tabela 5). 
Tabela 5 - Comparação das médias nas faixas etárias acima de 60 anos e abaixo de 60 anos das variáveis FPP D e E, MRC (MMSS, MMII e TOTAL) através do Teste T.

\begin{tabular}{llllll}
\hline \multirow{2}{*}{ FPP (D) } & Média & $\begin{array}{l}\text { Desvio } \\
\text { Padrão }\end{array}$ & $\begin{array}{l}\text { Erro } \\
\text { Médio }\end{array}$ & P \\
\multirow{2}{*}{ FPP (E) } & Menor que 60 & 25.26 & 6.847 & 1.428 & 0.000 \\
\multirow{3}{*}{ MRC (MMSS) } & Maior igual a 60 & 17.50 & 8.200 & 0.960 & \\
\cline { 2 - 6 } & Menor que 60 & 22.85 & 9.819 & 2.047 & 0.001 \\
MRC (MMII) & Maior igual a 60 & 15.51 & 8.577 & 1.004 & \\
\cline { 2 - 6 } & Menor que 60 & 26.52 & 3.964 & 0.827 & 0.003 \\
TOTAL (MRC) & Maior igual a 60 & 23.29 & 4.641 & 0.543 & \\
\cline { 2 - 6 } & Menor que 60 & 24.43 & 4.357 & 0.909 & 0.242 \\
\cline { 2 - 6 } & Maior igual a 60 & 23.18 & 4.492 & 0.526 & \\
\hline
\end{tabular}

* Comparação através do Teste T. Valor de $\mathrm{p} \leq 0.05$. MRC: Medical Research Council; MMII: Membros inferiores; MMSS: Membros superiores; FPP: Força de Preensão Palmar; Fonte: Autores (2019).

\section{Discussão}

Os achados sociodemográficos dos pacientes inclusos no presente estudo e admitidos no serviço hospitalar são consistentes com o estudo de Chalmers et al., (2010), no que se refere à prevalência de idosos, motivos da internação e comprometimento funcional, mesmo a sendo a causa da internação por motivos respiratórios.

Entre os diagnósticos que justificaram a internação, o fator infeccioso representado pela pneumonia, foi predominante (Chalmers et al., 2010). Segundo Bahlis et al., (2018) a prevalência da pneumonia vem aumentando no Brasil especialmente nas faixas etárias de 35 a 86 anos nos grupos vulneráveis, sendo esta, em sua maioria, de origem viral e bacteriana.

No que se refere à média de idade e vida sedentária, pesquisa anterior ao presente estudo, desenvolvido por Glebson et al., (2014), realizado no mesmo hospital, também identificou a prevalência de idosos com hábitos sedentários (80\% dos pacientes entrevistados). Desta forma, uma condição de sedentarismo, em indivíduos idosos, contribui para um estado de perda funcional que certamente será exacerbado no período de hospitalização, especialmente se for considerado que estes pacientes, desde quando admitidos já apresentavam menores médias de força muscular e funcionalidade.

Diversos estudos confirmam perda de força, funcionalidade e qualidade de vida em 57, 3\% dos pacientes internados por pneumonia. No presente estudo, os resultados obtidos através da MIF e do WHODAS demonstram que o comprometimento funcional já foi constatado nos primeiros dias de internação (Veiga et al., 2016). A média da MIF denotou estado de dependência modificada, onde a maioria dos pacientes necessitavam de assistência em até $25 \%$ das suas tarefas cotidianas. Este resultado é corroborado por Mitani et al., (2018), que utilizaram a MIF com o objetivo de avaliar a perda de funcionalidade de pacientes internados por pneumonia, afirmando que a maioria dos pacientes internados dependia de apoio durante atividades.

A média da escala WHODAS 2.0, demonstrou que a população estudada apresenta dificuldade moderada no desempenho de suas atividades. Isto se assemelha ao que foi visto no estudo realizado por Silva et al., (2016), que avaliaram o nível de incapacidade funcional de pacientes com Doença Pulmonar Obstrutiva Crônica (DPOC). O respectivo estudo identificou que os pacientes apresentaram leve incapacidade funcional, concluindo que indivíduos com DPOC, apresentam moderada a leve incapacidade no desempenho de suas atividades, desde mobilidade até sua participação social. É correto considerar que o fato de estarem hospitalizados seja uma condição que interfira no estado funcional, principalmente se forem 
considerados fatores como o estado ativo da doença, bem como o fato destes pacientes terem sido avaliados em data próxima à admissão ou no início do tratamento medicamentoso.

Os pacientes recém-admitidos com doenças respiratórias agudas e crônicas agudizadas já apresentavam fraqueza muscular periférica, fato confirmado tanto em relação ao MRC quanto em relação à FPP D e E. Sendo assim, sabe-se que a perda de força muscular para MMSS estabelece relação com a capacidade ventilatória da mesma forma que a força muscular de MMII estabelece relação com a funcionalidade. Estes dados se assemelham ao do estudo realizado por Nagato et al., (2012), que verificou que a força dos MMSS e a força de preensão palmar (FPP) estão relacionadas a alguns músculos que participam no posicionamento dos braços, possuindo ainda funções posturais e ventilatórias, estreitando assim, a relação da FPP com a musculatura periférica e a capacidade ventilatória nestes indivíduos.

A consciência de que estes pacientes já são admitidos em condição de fraqueza muscular ressalta a necessidade de adoção de acompanhamento fisioterapêutico motor sistemático e eficaz, que permita minimizar, controlar ou retardar a perda de força muscular, assim que possível (Oliveira et al., 2018). A abordagem fisioterapêutica deverá estar pautada na adoção de técnicas que aumentem força, resistência, controle e qualidade da função muscular. A partir disso, intervenções como os exercícios aeróbicos, podem aumentar a força muscular e a funcionalidade dos pacientes internados, reduzindo, consequentemente, a mortalidade (durante e após internação) e os índices de readmissão hospitalar (Calegari et al., 2017).

Verlaan et al., (2017) em seu estudo que utilizou a dinamometria para analisar a força muscular periférica de idosos durante o período de hospitalização e, a partir desta avaliação, notou-se que a redução da força muscular estava diretamente relacionada com o processo de internação e com uma menor probabilidade de sobrevivência após alta hospitalar. No atual estudo, houve redução significativa da força muscular de preensão palmar e da força muscular global, demonstrando que a piora do desempenho da musculatura periférica e dos músculos respiratórios correlacionam-se com a diminuição da capacidade aeróbia e da funcionalidade (Costa et al., 2021; Olguín et al., 2017).

Além dos fatores citados anteriormente, a falta de mobilização durante a hospitalização contribui decisivamente para as consequências do descondicionamento, levando a modificações da coordenação, do equilíbrio e da força muscular, por conseguinte alteração da funcionalidade motora (Silva et., 2008; Silva et al., 2008; Favarin; Camponogara, 2012). A hospitalização prolongada está associada diretamente à fraqueza muscular e ao descondicionamento físico, que afetam pacientes internados devido ao imobilismo. Assim, durante a restrição ao leito, pode ocorrer $30 \%$ de diminuição da força muscular, além de alterações nas fibras musculares, atrofia da musculatura periférica e respiratória (White; Wilson; Keysor, 2011). Logo, quaisquer avaliações validadas para se obter resultados citados nos itens supracitados são de fundamental importância para se determinar o perfil funcional e clínico de pacientes hospitalizados.

Por ser um estudo transversal, não foi possível estabelecer casualidade entre as variáveis. Alguns pacientes inclusos possuíam algumas comorbidades como Hipertensão arterial sistêmica (HAS), Diabetes Mellitus (DM), ou Insuficiência Renal Crônica (IRC), o que pode ter interferido nas medidas obtidas. Outro viés presente foi a falta da classificação da gravidade da DPOC nos pacientes que participaram da atual pesquisa.

\section{Conclusão}

Portanto, considerando a população estudada, houve prevalência de idosos, sedentários e fumantes entre os pacientes admitidos por afecções respiratórias, sendo a pneumonia o diagnóstico clínico com maior prevalência. Mesmo no período inicial da admissão, foi constatada redução significativa da força muscular periférica e respiratória, com significância estatística quando comparado com o predito, demonstrando ainda, correlações e interferências fortes, significativas entre as variáveis pesquisadas. Desta forma, os participantes deste estudo apresentaram uma redução moderada do estado funcional, apesar do motivo da internação não ser necessariamente motor (neurológico ou ortopédico). 
Nesse contexto, é necessário que futuros estudos sejam realizados com o objetivo de analisar as percas físicas e funcionais em pacientes hospitalizados, acompanhando assim, as diversidades das doenças, seus estágios e os seus desfechos clínicos.

\section{Referências}

Assis, C. S., Batista, L. C., Wolosker, N., Zerati, A. E., \& Silva, R. C. G. (2015). Medida de independência funcional em pacientes com claudicação intermitente. Rev Esc Enferm USP, 49(5), 756-761.

Bahlis, L. F., Diogo, L. P., Kuchenbecker, R. S., \& Fuchs, S. C. (2018). Perfil clínico, epidemiológico e etiológico de pacientes internados com pneumonia adquirida na comunidade em um hospital público do interior do Brasil. J. bras. Pneumol, 44(4), 261-266.

Basic, D., Chróinín, D. N., Conforti, D., \& Shanley C. (2017). Predictors on admission of functional decline among older patients hospitalised for acute care: A prospective observational study. Australasian Journal on Ageing, 36(4), 1-7.

Calegari, L., Barroso, B. F., Bratz, J., Romano, S., Figueiredo, G. F., Ceccon, M., Pimentel, G. L., \& Reolão, J. B. C. (2017). Efeitos do treinamento aeróbico e do fortalecimento em pacientes com insuficiência cardíaca. Rev Bras Med Esporte, 23(2), 123-127.

Chalmers, J. D., Singanayagam, A., Akram, A. R., Mandal, P., Short, P. M., Choudhury, G., Wood, V., \& Hill, A. T. (2010). Severity assessment tools for predicting mortality in hospitalised patients with community-acquired pneumonia: systematic review and meta-analysis. Thorax, 65(10), 878-883.

Coker, R. H., Hays, N. P., Williams, R. H., Wolfe, R. R., \& Evans, W. J. (2015). Bed rest promotes reduction in walking speed, functional parameters, and aerobic fitness in older, healthy adults. J Gerontol A Biol Sci Med Sci, 70(1), 91-96.

Costa, A. L. S. N., Rego, R. S., Sousa, A. M., França, R. G. O., Magalhães, B. C., \& Araújo, C. G. B. (2021). Correlação da força de preensão palmar e estado nutricional em idosas praticantes de atividade física. Research, Society and Development, 10(5), 1-12.

Favarin, S. S., \& Camponogara, S. (2012). Perfil dos pacientes internados na unidade de terapia intensiva adulto de um hospital universitário. Rev Enferm UFSM, 2(2), 320-329.

Federici, S., Bracalenti, M., Meloni, F., \& Luciano, J. V. (2017) World Health Organization Disability Assessment Schedule 2.0: an international systematic review. Disabil Rehabil, 39(23), 2347-2380.

Ferreira, R., Neuparth, M. J., Ascensão, A., \& Magalhães, J. (2004). Atrofia muscular esquelética. Modelos experimentais, manifestações teciduais e fisiopatologia. Rev Portug Ciênc Desp, 4(3), 94-111.

Ferreira, V. D., Cunha, T. M., Silva, I. S., Nogueira, P. A. M. S., \& Fonseca, A. M. C. (2018). Relação entre força muscular periférica e funcionalidade em pacientes críticos. ConScientiae Saúde, 17(3), 315-321.

Figueiredo, I. M., Sampaio, R. F., Mancini, M. C., Silva, F. C. M., \& Souza, M. A. P. (2007). Teste de força de preensão utilizando o dinamômetro Jamar. Acta Fisiatr., 14(2), 104-110.

Lima-Costa, M. F., Loyola-Filho, A. I., \& Matos, D. L. (2007). Tendências nas condições de saúde e uso de serviços de saúde entre idosos brasileiros: um estudo baseado na Pesquisa Nacional por Amostra de Domicílios (1998, 2003). Cad Saúde Pública. 23(10), 2467-2478. < https://www.arca.fiocruz.br/handle/icict/6619>.

Martinez, B. P., Batista, A. K. M. S., Gomes, I. B., Olivieri, F. M., Camelier, F. W. R., \& Camelier, A. A. (2015). Frequence of sarcopenia and associated factors among hospitalized elderly patients. BMC Musculoskelet Disord, 16(108), 2-7.

Morosini, S., Marques, A. P. O., Leal, M. C. C., Marino, J. G., \& Melo, H. M. A. (2011). Custo e tempo de permanência hospitalar de idosos residentes em Recife - PE. Geriatria \& Gerontologia, 5(2), 91-8.

Nagato, A., Barboza, C. R., Manso, R. G., Oliveira, M. F., Silva, M. A., \& Bezerra, F. S. (2012). Influência do posicionamento dos membros superiores sobre parâmetros ventilatórios em indivíduos adultos. Fisioter. Mov., 25(3), 525-532.

Neder, J. A., Andreoni, S., Lerario, M. C., \& Nery, L. E. (1999). Reference values for lung function tests: II. Maximal respiratory pressures and voluntary ventilation. Braz J Med Biol Res, 32(6), 719-727.

Olguín, T., Bunout, D., Pía de la Maza, M., Barrera, G., \& Hirsch, S. (2017). Admission handgrip strength predicts functional decline in hospitalized patients. Clinical Nutrition ESPEN, 17, 28-32.

Oliveira, M. F., Santos, R. C., Artz, S. A., Mendez, V. M. F., Lobo, D. M. L., Correira, E. B., Ferraz, A. S., Umeda, I. I. K., \& Sperandio, P. A. (2017). Segurança e eficácia do treinamento aeróbio combinado à ventilação não-invasiva em pacientes com insuficiência cardíaca aguda. Arq. Bras. Cardiol., 110(5), 467-475.

Organização Mundial da Saúde. Avaliação de saúde e deficiência: manual do WHO Disability Assessment Schedule 9 (WHODAS 2.0). Uberaba: Universidade Federal do Triangulo Mineiro; 2015 [citado 7 ago 2017]. <http://apps.who.int/iris/ bitstream/10665/43974/19/9788562599514_por.pdf >.

Romanholi-Cória, V., Grigolo, I. H., Mutter, V. M., Defende, G. S., Moras, L. L., Morales, S. T., Carrai, L. H. M., Dias, A. L. O., Costa, N. M., Budin, A. A., Rodrigues, C. D., Marchiori, R. B., Sasso, D. T., Calocini, A. G., Lima, J. C., Reis, A. F. N., Kuyumjian, F. G., \& Cação, J. C. (2017). Caracterização dos idosos internados por doença respiratória aguda em um hospital escola terciário. Revista De Medicina, 96(2), 94-102.

Santos, M. I. P. O. (2007). Perfil dos idosos internados no hospital geral em Belém (Pará). Esc Anna Nery R Enferm, 11(1), 23-29. 
Research, Society and Development, v. 10, n. 14, e538101422426, 2021

(CC BY 4.0) | ISSN 2525-3409 | DOI: http://dx.doi.org/10.33448/rsd-v10i14.22426

Silva, C. M. S., Pinto Neto, A. C., Nepomuceno Júnior, B. R. V., Teixeira, H. P., Silveira, C. D., \& Sousa-Machado A. (2016). Incapacidade funcional de pacientes com Doença Pulmonar Obstrutiva Crônica (DPOC) através do WHODAS. Acta Fisiatr., 23(3), 125-129.

Silva, G. M., \& Menezes, G. G. S. (2014). Avaliação do perfil sócio demográfico e hábitos de vida dos pacientes hospitalizados no munícipio de Lagarto, Sergipe. Scientia Plena, 10(3), 1-9.

Silva, K. R., Marrara, K. T., Marino, D. M., Di Lorenzo, V. A. P., \& Jamami, M. (2008). Fraqueza muscular esquelética e intolerância ao exercício em pacientes com doença pulmonar obstrutiva crônica. Revista Brasileira de Fisioterapia, 12(3), 169-175.

Silva, M. R., Anzolin, R. M., Claro, T. C., \& Medeiros, T. C. (2008) Efeitos deletérios: ausência da cinesioterapia na mobilidade articular em politraumatizado. Fisioter. Mov., 21(2), 39-45.

Veiga, B., Pereira, R. A. B., Pereira, A. M. V. B., \& Nickel R. (2016). Avaliação de funcionalidade e incapacidade de idosos longevos em acompanhamento ambulatorial utilizando a WHODAS 2.0. Rev. Bras. Geriatr. Gerontol., 19(6), 1015-1021.

Verlaan, S., Van Ancum, J. M., Pierik, V. D., Van Wijngaarden, J. P., Scheerman, K., Meskers, C. G. M. \& Maier, A. B. (2017). Muscle measures and nutritional status at hospital admission predict survival and independent living of older patients - the empower study. J Frailty Aging, 6(3), 161-166.

Vieira, R. H. G., Nogueira, I. D. B., Queiroz, N. F., Cunha, T. M., Araújo, Z. T. S., Vieira, W. H. B., \& Nogueira, P. A. M. S. (2018). Força muscular periférica e respiratória na Doença Pulmonar Obstrutiva Crônica. Rev. bras. cineantropom. desempenho hum, 20(2), 125-133.

Volianitis, S., McConnell, A. K., Koutedakis, Y., \& Jones, D. A. (2001). Specific respiratory warm-up improves rowing performance and exertional dyspnea. Med Sci Sports Exerc, 33(7), 1189-1193.

White, D. K., Wilson, J. C., \& Keysor, J. J. (2011). Measures of adult general functional status: SF-36 Physical Functioning Subscale (PF-10), Health Assessment Questionnaire (HAQ), Modified Health Assessment Questionnaire (MHAQ), KATZ Index of Independence in activities of daily living, Functional Independence Measure (FIM), and Osteoarthritis-Function-Computer Adaptive Test (OAFunction-CAT). Arthritis Care Res (Hoboken), Supp1 11:S297-307. 